\title{
A multi-user decision support system for online city bus tour planning
}

\author{
Saeed Asadi Bagloee ${ }^{1}$ Madjid Tavana ${ }^{2,3}$ - Debora Di Caprio ${ }^{4,5}$. \\ Mohsen Asadi ${ }^{6} \cdot$ Mitra Heshmati $^{7}$
}

Received: 19 October 2016/Revised: 4 March 2017/Accepted: 11 March 2017/Published online: 31 March 2017

(C) The Author(s) 2017. This article is an open access publication

\begin{abstract}
Tourism is rapidly becoming a sustainable pathway toward economic prosperity for host countries and communities. Recent advances in information and communications technology, the smartphone, the Internet and Wi-Fi have given a boost to the tourism industry. The city bus tour (CBT) service is one of the most successful businesses in the tourism industry. However, there exists no smart decision support system determining the most efficient way to plan the itinerary of a CBT. In this research, we report on the ongoing development of a mobile application (app) and a website for tourists, hoteliers and travel agents to connect with city bus operators and book/purchase the best CBT both in terms of cost and time. Firstly, the CBT problem is formulated as an asymmetric sequential three-stage arc routing problem. All places of interest (PoI) and pickup/dropout points are identified with arcs of the network (instead of nodes), each of which can be visited at least once (instead of exactly
\end{abstract}

Madjid Tavana

tavana@lasalle.edu;

http://tavana.us/

Saeed Asadi Bagloee

saeed.bagloee@unimelb.edu.au

Debora Di Caprio

dicaper@mathstat.yorku.ca; debora.dicaprio@istruzione.it

Mohsen Asadi

mohsenasadibagloee@yahoo.com.au

Mitra Heshmati

Mitra.Heshmati@monash.edu

1 Department of Infrastructure Engineering, Melbourne School of Engineering, University of Melbourne, Parkville, VIC, Australia once). Secondly, the resulting pure integer programming (IP) problem is solved using a leading optimization software known as General Algebraic Modeling System (GAMS). The GAMS code developed for this project returns: (1) the exact optimal solution identifying the footprints of the city bus relative to all the arcs forming the minimal cost network; (2) the augmenting paths corresponding to the pickup stage, the PoI visiting stage and the drop-off stage. Finally, we demonstrate the applicability of the mobile app/website via a pilot study in the city of Melbourne (Australia). All the computations relative to the initial tests show that the ability of the app to answer users' inquiries in a fraction of a minute.

Keywords Decision support system · Tourism · Operations planning - Information and communications technology
2 Business Systems and Analytics Department, Distinguished Chair of Business Analytics, La Salle University, Philadelphia, PA 19141, USA

3 Business Information Systems Department, Faculty of Business Administration and Economics, University of Paderborn, 33098 Paderborn, Germany

4 Department of Mathematics and Statistics, York University, Toronto M3J 1P3, Canada

5 Polo Tecnologico IISS G. Galilei, Via Cadorna 14, 39100 Bolzano, Italy

6 Department of Civil and Environmental Engineering, Kharazmi University, Tehran, Iran

7 Faculty of Engineering, Monash University, Clayton, SA, Australia 


\section{Introduction}

Tourism is rapidly becoming a sustainable pathway toward economic prosperity for host countries and communities. Recent advances in information and communications technology (ICT), the smartphone, the Internet and Wi-Fi have given a boost to the tourism industry [1-5].

In the last thirty years, the energetic growth and development of the tourism industry has been mirrored by the growth of ICT [6], which adds value to tourism services and products throughout the value chain. Nowadays, all tourism-related activities, from hotel reservations and air tickets to visa applications, are pre-planned online (see Fig. 1).

The capacity of mobile technology in assisting travelrelated decisions has substantially improved with respect to that offered by the early prototype of mobile tour-guides. From GPS-supported systems and camera phones to today's smartphones with enhanced information and communication capabilities [7], we have witnessed an ICT escalation that has provided a very fertile ground to promote tourism.

Sightseeing in cities is an indispensable part of almost all tourists' plans. A large body of research is dedicated to identify the most attractive places and major landmarks for tourists to visit. Once identified, these places, usually referred to as places of interest (PoI), need to be reached and visited by the tourists, which is the problem we focus on in this study. City bus tour (CBT) service, that is, a specific bus service providing pre-planned tours to a number of PoI, is an alternative commonly preferred to public transportation. Thus, the problem becomes the design of CBT routes that allow the tourists to save time while reducing the operational costs of the city bus company.

Due to the surge of interest shown by experts of different disciplines, city tour planning tools have proliferated, incorporating an array of useful services [8]. However, the existing tools still fail to address several practical aspects, hence compromising their utility in realistic tourist scenarios [9].

Formally speaking, the problem belongs to the class of minimum cost network flow problems $[10,11]$ that are in general intractable (NP-hard) and involve optimal and/or heuristic algorithms and methods characterized by different types of time complexity [12-19]. This is particularly true when dealing with networks of extremely large size. Note that while this can also be the case of urban networks, the road networks connecting PoI are usually small in size or composed of small size blocks.

Thus, the main issue is to formulate a mathematical model that makes the problem tractable. From a practical viewpoint, the goal is to guarantee that a solution (minimum cost flow) exists and can be obtained using a specific optimization software with relatively good performance (time complexity: reasonably fast). This would open the way to the development of a mobile applications (app) capable of addressing the tourists' need of visiting a given

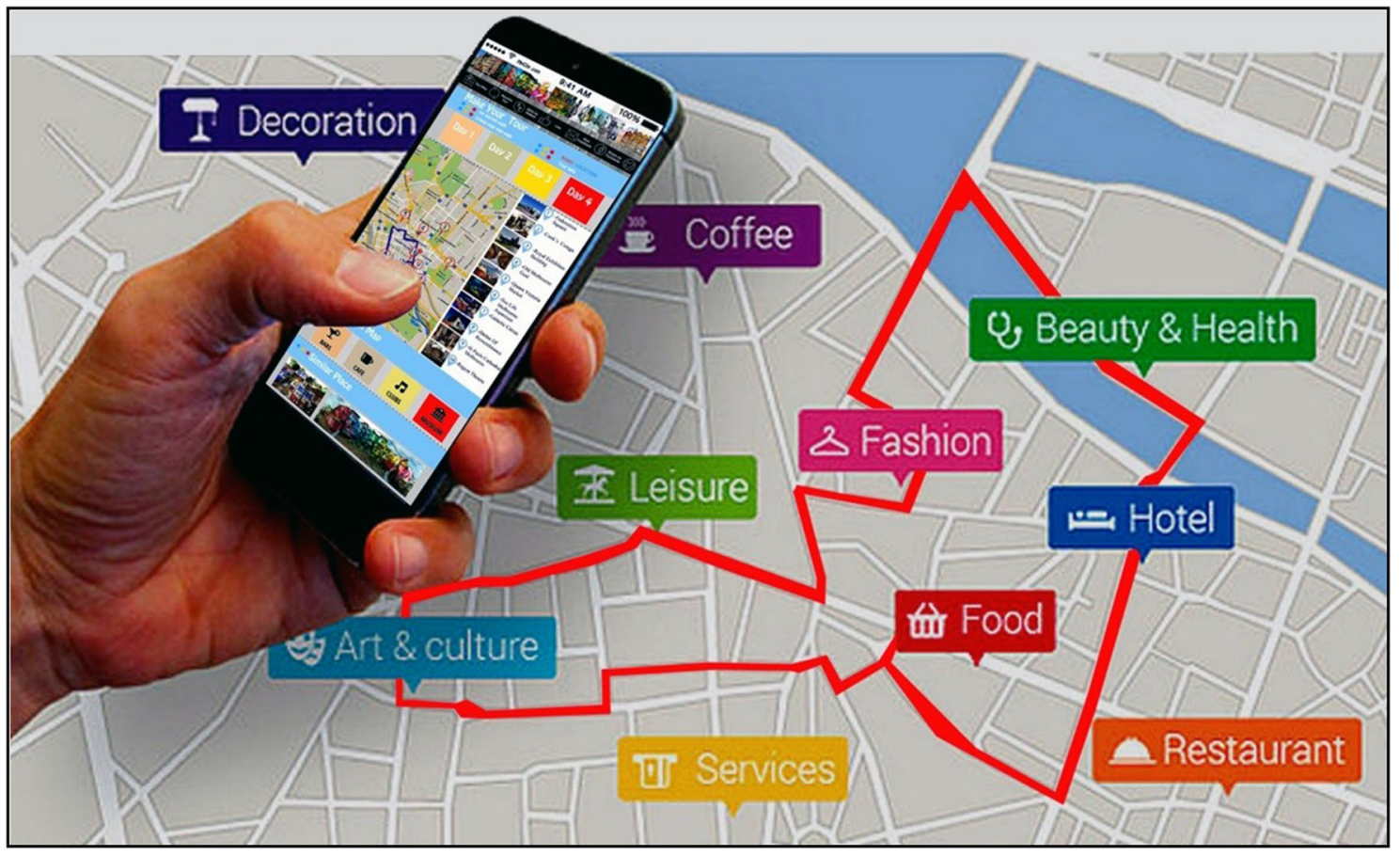

Fig. 1 Tourism industry and ICT (Internet, mobile communication) 
number of PoI in the shortest possible time period using a CBT service.

In this study, we propose an efficient decision support system (DSS) for connecting city bus companies to hoteliers, travel agents and tourists. The results presented are part of an extensive collaboration between the University of Melbourne (Australia), La Salle University (United States) and York University (Canada). The aim is to develop a user-friendly mobile app through which endusers or customers (here the tourists or agents) can book and purchase their favorite tour plan.

An app is a computer program that is written and based on the Android operating system to run on mobile phones. Empirical evidence indicates a preference for mobile apps over other ICT means including websites [7, 20, 21]. Nevertheless, the software is usually also made available online via a traditional website interface to cater to hoteliers, travel agencies and tour operators and enables them to book in bundles for their own guests or customers.

Figure 2 provides a screenshot of the app/website being developed for booking the optimal CBT, while Fig. 3 shows a schematic view of the architecture of the proposed DSS.

The mathematical formulation of the CBT problem under consideration is an arc routing variant of the sequential formulation of the Traveling Salesman Problem (TSP) proposed by [22]. More precisely, our formulation differs from a classical TSP in four main aspects:
1. Both the PoI and the pickup/dropout points are identified with arcs of the network instead that with nodes (i.e., an arc routing problem is considered);

2. We relax the typical "visiting restriction" stating that each PoI must be visited exactly once by requiring that each PoI is to be visited at least once;

3. All our variables are assumed to take integer values (i.e., there is no continuous variable);

4. We allow for the cost associated with a link to change according to how the link is directed (i.e., an asymmetric and, hence, more general variant of TSP is considered).

Moreover, we model the problem under the assumption that the CBT service uses only one bus $(q=1)$. Hence, we obtain an integer programming problem with binary decision variables (i.e., a directed arc of the network flow cannot be traversed more than once). An extension of the current study could be made considering the fleet size $(q>1)$.

Our approach merges and simultaneously addresses two main issues related to arc routing problems: identifying the footprints of a minimum cost network flow and augmenting the corresponding residual network in search of minimal flows. The fact that this twofold problem can be modeled as a customary and relatively simple pure integer programming (IP) problem for which a feasible path-based solution always exists is one of the merits of this study.

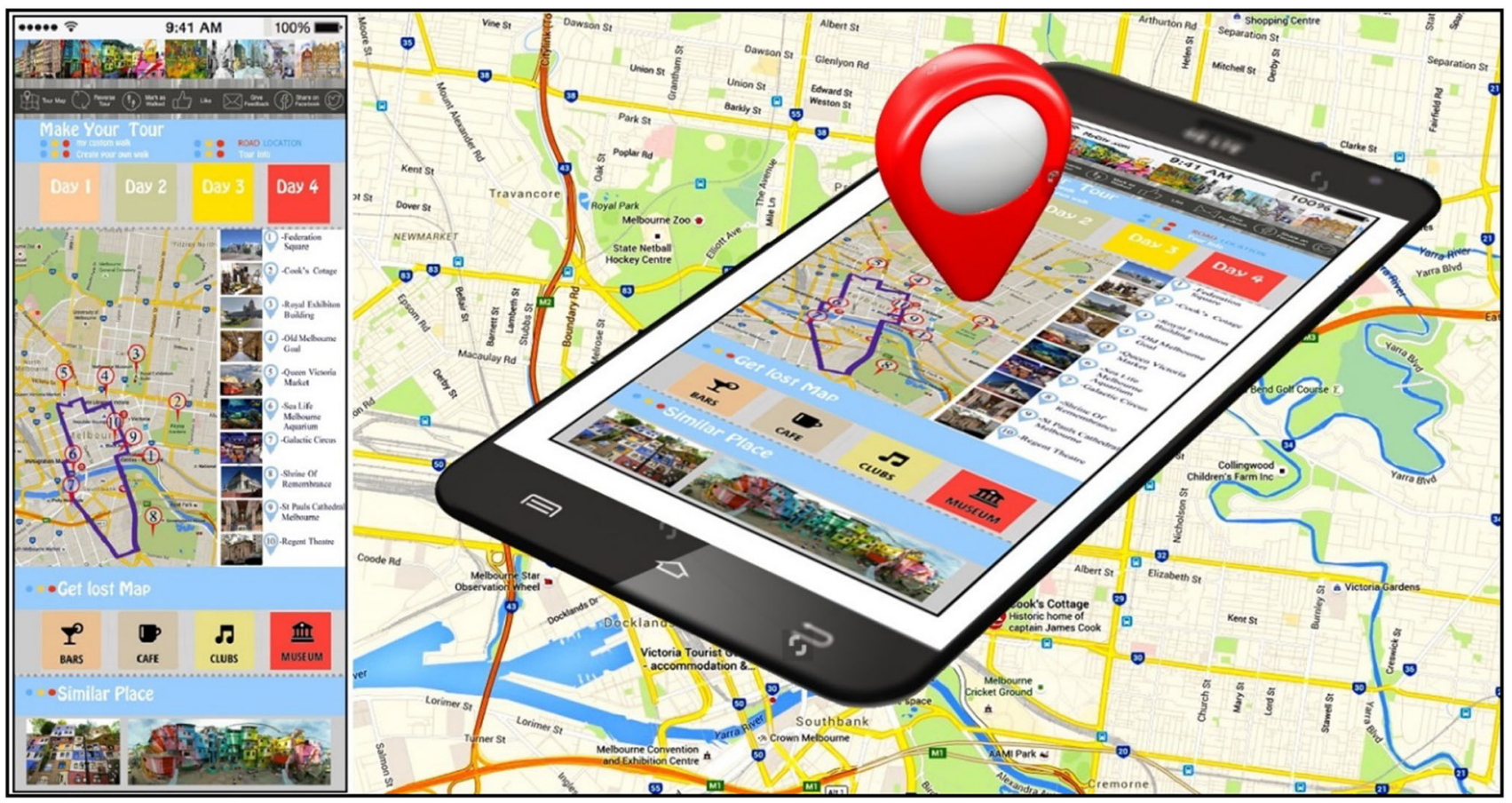

Fig. 2 Screenshots of the app/website being developed for city bus booking 


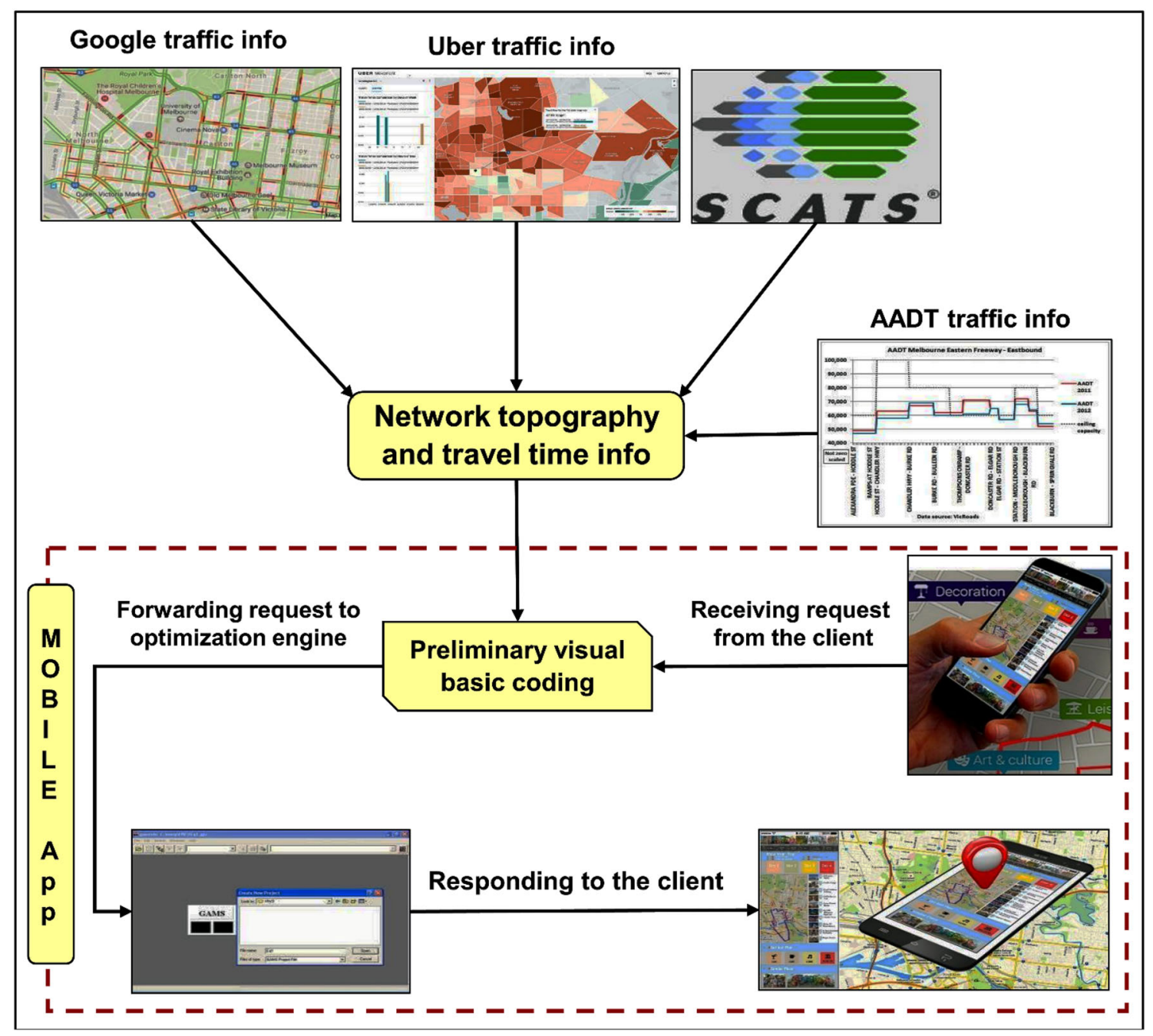

Fig. 3 A schematic view of the architecture of the decision support system (DSS)

Another merit of the current study is the specific purpose optimal method introduced to solve the proposed IP. This method allows finding an exact optimal solution to the IP problem by implementing an optimization software with no need to introduce advanced heuristic methods. More precisely, we managed integrating different kinds of software working on a single platform, namely the optimization platform of the General Algebraic Modeling System (GAMS), developing a code that returns the solution to the proposed real-life problem in a fraction of a minute.

Due to commercial-related issues, the designed mobile app has not been lunched yet, and thus, we cannot provide the corresponding website. Nevertheless, we can demonstrate the applicability of the mobile app/website presenting a pilot study performed in the city of Melbourne (Australia). The GAMS code developed for this project is available in "Appendix."

The remainder of this paper is organized as follows. In Sect. 2, we discuss some of the recent literature on the influence of ICT on the tourism industry elaborating, in particular, on tourists' travel planning and sightseeing in cities. In Sect. 3, we describe the general characteristics of a CBT service and its interpretation as a three-stage arc routing problem. In Sect. 4, we introduce the mathematical model and the methodology behind the mobile app of the proposed DSS. In Sect. 5, we present the results of a pilot study conducted in the city of Melbourne (Australia). Finally, in Sect. 6, we present our conclusion and outline some future lines of research.

\section{Literature review}

\subsection{ICT and tourists' travel planning}

ICT has become further woven into the fabric of our daily life in areas such as leisure, entertainment and travel $[23,24]$. The Internet and the evolution of the mobile 
phone into the smartphone have created an especially powerful ICT tool in tourism and destination management, primarily because it facilitates the transmission of communication and information quickly and inexpensively $[24,25]$. Consumers search the Internet for travel information in order to reduce risk and uncertainty before making tourism purchases [26]. At the same time, stakeholders in the tourism industry, such as airlines, hotels and attractions, have developed services that allow customers to interact through mobile channels [27, 28]. For a comprehensive review of the recent developments in tourism and hospitality in light of communication technologies, the reader may refer to [29].

According to recent estimates, more than $73 \%$ of the population of all ages in the USA is using smartphones [30]. This figure is even higher in Australia with about 80\% of the population using smartphones [31]. Moreover, the market penetration of the smartphone across the world is on the rise [25, 32, 33]. Such a pervasive usage of the mobile and smartphone technology has created a totally new paradigm in the tourism business and opened the way to a completely untapped market to be exploited. In particular, mobile bookings are on the rise at an exponential pace [32]. The mobile travel market value in the USA reached US $\$ 23$ billion in 2013 with a $16 \%$ increase compared to the previous year [21]. A similar trend can be observed in the European travel market [20, 34].

Many hotels and online travel agencies wish to be at the forefront of this trend in the adoption of smartphones for travel planning and hospitality services [21]. It is expected that the smartphone, along with the mobile platform, will play an important role not only in the distribution of products, but also in establishing and strengthening customer relationships and brand loyalty [21, 34].

\subsection{Sightseeing}

Sightseeing in cities is an essential part of travel planning and there already exists a considerable amount of studies in the literature focusing on how to select the PoI that tourists should visit.

Flagging the most important PoI (among many) and establishing their time-sequencing combine in a particularly laborious task requiring the skilled interaction of a multitude of resources $[35,36]$. However, decisions on what places to include among the PoI are largely made based on tourists' preferences, often overlooking the difficulties that one may have to get there (transportation) [37-42].

At the same time, when it comes to transport models, tourists are especially reluctant to use public transportation services, mainly because they feel that they do not have the knowledge to move through the local systems or negotiate with the local people efficiently. Furthermore, tourists also consider the risk of leaving the tourism space and entering terra incognita should they use the wrong service or take the wrong direction [43].

On a related matter, in the last decades, regret has been increasingly indicated as a common denominator in many practical and theoretical issues addressed by the literature on travel and city tour planning. Among the most recent works related to transportation and logistics management [44] offer a comparative empirical study of random regret minimization (RRM) as a complementary modeling paradigm to random utility maximization (RUM). They focus on the behavioral differences in willingness to pay estimates, choice elasticities and choice probabilities that arise when accounting for the regret of not choosing the "best" alternative. Similar studies and overviews have been proposed, among others, by [45-48]. Finally, several recent studies show an approach to travelers' decision behavior modeling more similar in spirit to a non-regrettable choice setting than to a risk/uncertainty one (see, for example, $[49,50])$.

The above discussion underlines the need for ICT tools to assist tourists in negotiating their way around various PoI, whether that will be walking or using public transit. Such tools typically appear in the form of personalized tourist guides that tackle a problem commonly termed the Tourist Trip Design Problem (TTDP) [51, 52]. A review of the applications of dynamic guided tours to provide realtime information to the tourists as they are exploring was provided by [53]. A more recent and comprehensive review on this line of research has been provided by [51].

\subsection{The city bus tour (CBT) alternative}

Several studies tend to offer the maximum number of PoI based on the availability of public transport without taking into consideration the stochastic component of the services such as bus/train/tram arrival times [9, 42]. The alternative to public transportation is given by a convenient and relatively affordable option known as city bus tour (CBT) service, that is, a specific bus service providing pre-planned tours to a number of PoI. These buses pass close by the major landmarks, while a pre-recorded or live commentary is played through headphones to passengers. Though such touristic schemes are not new, they are becoming more popular among the travelers who are in turn becoming more independent, experienced, sophisticated, demanding and harder to please $[1,5,54]$.

Typically, city bus tickets are issued for one- or two-day periods. Tickets are either purchased at tourist attractions or online. The market share of the CBT services is staggering and growing exponentially. For example, the annual rides of a well-known international company providing 
CBT services and operating in approximately 30 cities across the world accounted for nearly 13 million tourists in 2011 [55]. The private sector is heavily involved in such a business which is the driving force behind the promotion of the tourism industry in general and the economy of the single cities in particular.

Given the stochastic nature of the CBT services mainly determined by variations of travel times (primarily due to traffic congestion), variations of tourist demands, limited availability of fleet, and high operating costs, a thriving city bus scheme must be designed and operated to the highest efficiency. Existing schemes are largely run based on the rule-of-thumb and field experience, with rigid timetables and itinerary plans. To the best of our knowledge, no study has so far been dedicated to this line of business.

\section{Interpreting CBT schemes as three-stage arc routing problems}

A CBT service usually operates as follows. A city bus company organizes a number of pre-planned tours based on its available fleet (i.e., the number of vehicles, $q$, that the company can use), the duration of the tours and the opening hours of the PoI (full-day or half-day) as well as seasonal factors and demands. The hoteliers and travel/tour agencies can go online and purchase CBTs subject to seat availability. Individual tourists can do the same using their mobile phone. The tourists are allowed to choose from a list of pickup/dropout designated places or to pay extra and request a pickup/dropout service at their own favorite spots.

Once the online purchase is made (either by agencies or individual tourists), the customers are notified of the approximate pickup/drop-off times pending a precise itinerary. The precise itinerary, including pickup/drop-off times, arrival times at $\mathrm{PoI}$ and lunch time are sent to the customer in less than an hour time. Tourists may leave the city bus and board again without a time limit (called "hop-on hop-off") at designated stops on a circle route as per the itinerary plan.

The key idea of these sorts of schemes is to provide tourists with an itinerary carefully tailored to satisfy their necessities and allow them to make their own decisions about destinations and services in the most flexible way and with little or no involvement of travel intermediaries [56].

In order to formulate the mathematical model and, consequently, look for a suitable software implementation that will allow the introduction of a mobile app to address the customers' requests, we consider the following setting.

A city bus (i.e., $q=1$ ) is located at a place called "depot" where requests from various corners of a city (i.e., the pickup points) are received. The journey of the city bus consists of three parts. In the first part of its journey, the city bus reaches each and every pickup point to collect tourists. Once all the tourists are on board, the city bus starts the second part of its journey bringing the tourists to visit a pre-specified number of PoI. In the third part, the city bus drives toward the dropout points which could be the same as the pickup points. Figure 4 demonstrates a typical tour (out of many) for an artificial map: The pickup and dropout points as well as the PoI and the depot are flagged. The three routes of the bus tour are also clearly shown in the figure. It is conceivable that there could be a myriad of such tours, so that the efficacy of the system depends upon finding the most efficient one. In this sense, the efficacy can be defined in terms of finding the tour with the least touring or travel time, that is, the tour that saves time to the tourists and lowers the operational costs of the city bus companies, simultaneously.

As set out above, the problem can be classified as a fleet management problem. The study of this kind of problems constitutes an active area of research in both academia and industry. The common sense approach is to encode the transportation network in a graph, that is, a set of nodes (or vertices) connected by arcs (or links, or roads).

The PoI and pickup/dropout points can be represented by either nodes or arcs (as shown in Fig. 4), which results in two different classes of problems. In the case where nodes are used for the representation, the problem belongs to the class of the node routing problems; if arcs are used, then the problem belongs to the class of the arc routing problems.

A classic example of node routing problem is the wellknown Traveling Salesman Problem (TSP) that generalizes to the so-called Vehicle Routing Problem (VRP), while the Chinese Postman Problem (also known as Postman Tour or Route Inspection Problem) is an example of arc routing problem.

In their conventional form, all these problems require each node/arc of a given set to be visited exactly once. We relax this restriction by requiring that each node/arc is visited "at least once." Our weaker assumption is justified by the fact that real-life road networks may be too poorly connected for each place of interest (PoI) or pickup/dropout point to be visited only once. In these cases, some nodes not only represent some of the PoI or pickup/dropout points, but also provide access to other PoI or pickup/dropout such as those located in dead-end alleys.

Consider, for instance, the simple one road network represented in Fig. 5. This figure illustrates a number of PoI placed on both sides of Little Bourke St between Elizabeth St and Queen St in Melbourne. The road connecting the PoI has been identified with the link $(1,2)$.

As already commented in the introduction, both types of problem are proven to belong to the class of the NP-hard problems, that is, highly difficult problems in terms of computational burdens and efficacy. 


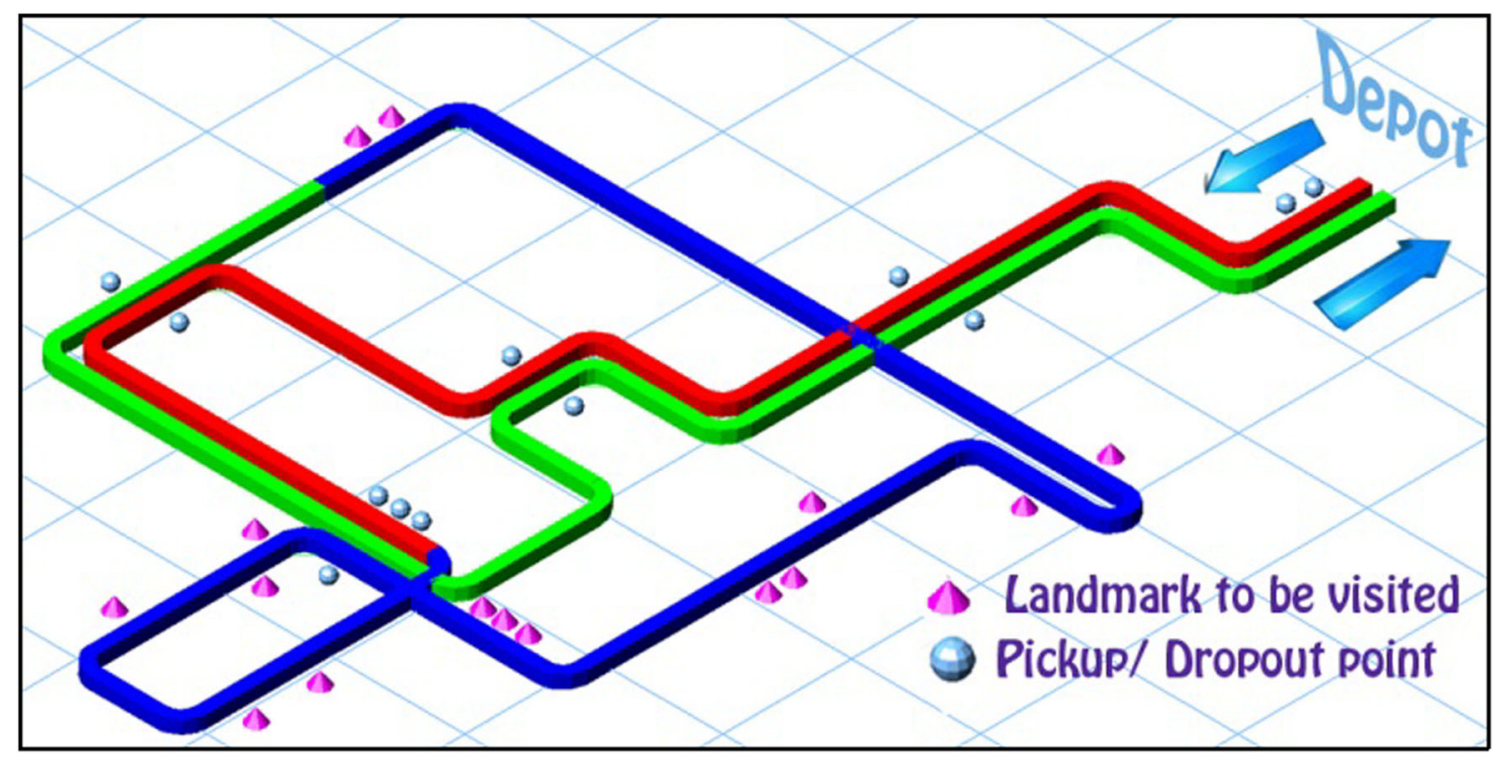

Fig. 4 A typical tour of a city bus in three stages: stage 1 (red colored) collecting tourists from pickup points, stage 2 (blue colored) visiting places of interest and stage 3 (green colored) dropping out tourists

Since computational efficacy is strictly related to the size of the network or graph (i.e., to the number of nodes and arcs), we represent the PoI and pickup/dropout points as links, as opposed to nodes. This considerably reduces the size of the network. For example, in Fig. 5, all the PoI marked are represented by links connected to the undirected link $(1,2)$.

Each link is associated with a cost that accounts for the traveling time (i.e., the time necessary to go through to the road represented by the link) and the visiting time (i.e., the time necessary to visit the PoI located on both sides of the road being considered). Accordingly, the itinerary of a CBT can be formulated as an optimization problem whose objective is the minimization of the total traveling time. Generally speaking, this problem can have very rich and diverse applications, such as robotic surveillance [18, 57], detection of forest fires [58], tourist trip planning [59] and police crime response [60].

In the next section, we provide the mathematical formalization of the itinerary of a city bus as a pure IP problem and describe the proposed optimal solution method. A pilot study conducted in the city of Melbourne to test the mobile app of the proposed DSS will be presented in Sect. 5.

\section{Mathematical model and solution method}

Let us represent the road network as a graph $G(N, A)$ where $N$ and $A$ are the set of nodes (or vertices) and the set of links (or arcs), respectively. Each road is identified with an undirected link connecting a node $i \in N$ to a different node $j \in N$ and denoted by a pair $(i, j) \in A$. That is, each road can be traversed in either one of the two ways possible, either in the direction $i \rightarrow j$ or in the direction $j \rightarrow i$.

For every $(i, j) \in A$, let:

- $x_{i j}$ denote the number of times that the city bus drives through the link $(i, j)$ in the direction $i \rightarrow j$;

- $\quad c_{i j}$ be the cost associated with the link $(i, j)$ when it is traversed in the direction $i \rightarrow j$.

The $x_{i j}$ 's are the decision variables of the optimization problem. The cost $c_{i j}$ accounts for the time needed to traverse the road $(i, j)$ in the direction $i \rightarrow j$, the visiting time of the corresponding PoI (if any), and the waiting times at pickup/dropout points (if any) for boarding or alighting. Note that $c_{i j}$ does not necessarily coincide with $c_{j i}$, that is, we consider an asymmetric arc routing problem, a more general variant of arc routing problem.

A city bus is deployed from a depot (or origin) node $o$, and once it has completed its tour, it heads to an exit or destination node denoted by $d$, which could also coincide with the depot node. Thus, the problem is finding the cheapest way of sending the city bus from $o$ to $d$ through the network $[61,62]$ while imposing that all the PoI and the pickup/dropout links are visited at least once.

Using the notations above and interpreting the distance covered by the bus when traveling through an arc (i.e., the length of the arc) as the cost of traversing the link itself, the IP formulation of the TSP problem proposed by [22] can be rewritten as follows:

$\min \sum_{(i, j) \in A} c_{i j} \times x_{i j}$, 


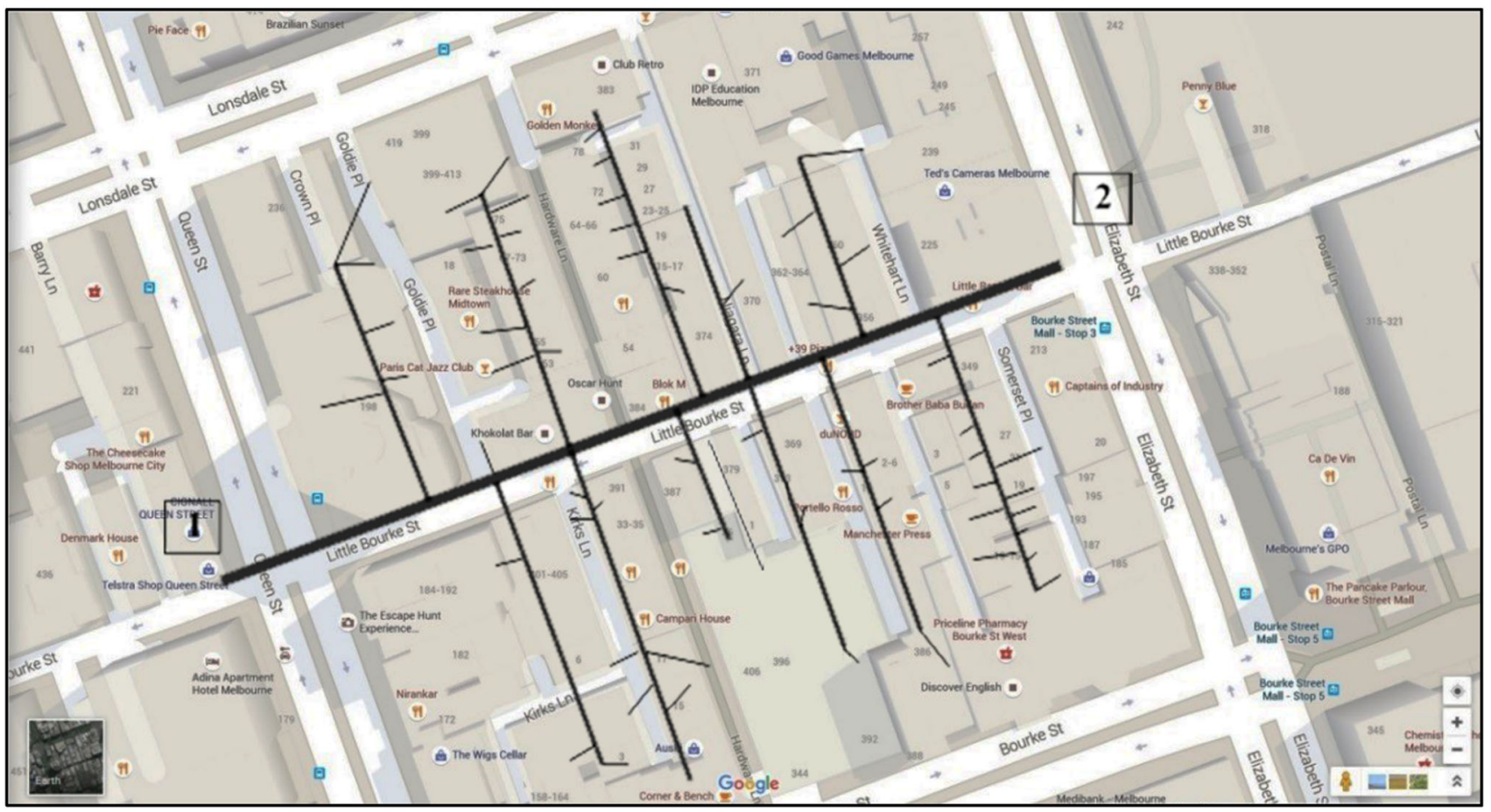

Fig. 5 Places of interest identified with links or nodes (map: part of Melbourne's downtown)

s.t.:

$\sum_{i \in N, i \neq j} x_{i j}=1 \quad \forall j \in N \backslash\{o, d\}$,

$\sum_{j \in N, j \neq i} x_{i j}=1 \quad \forall i \in N \backslash\{o, d\}$,

$y_{i}-y_{j}+|N| \times x_{i j} \leq|N|-1 \quad \forall i, j \in N \backslash\{o, d\}, \quad i \neq j$,

$x_{i j}$ non-negative integer $\forall(i, j) \in A$,

$y_{i}$ real number $\forall i, j \in N \backslash\{o, d\}$,

where $o=d$ and, $\forall(i, j) \in A, c_{i j}=c_{j i}=$ lenght of $(i, j)$.

As observed by [22], the constraints necessarily imply that $\forall(i, j) \in A, x_{i j} \in\{0,1\}$, while it is permissible to restrict the variables $y_{i}$ to take nonnegative integers.

In the above formulation, the objective function of Eq. (1) minimizes the total traveling time. Constraints (2) and (3) represent the condition that each node (other than $o$ ) is visited exactly once, while Constraint (4) guarantees that all feasible flows start at $o$ and end at $d$ eliminating the possibility of any sub-tour.

A standard IP formulation that generalizes the one proposed by [22], i.e., Eqs. (1) to (6) are as follows (see, for instance, $[63,64])$ :

$\min \sum_{(i, j) \in A} c_{i j} \times x_{i j}$ s.t.:

$$
\begin{gathered}
\sum_{(i, n) \in \delta^{+}(i)} x_{n j}-\sum_{(i, n) \in \delta^{-}(i)} x_{i n}= \begin{cases}-1 & \text { if } n=d \\
+1 & \text { if } n=o \\
0 & \text { if } n \in N \backslash\{o, d\}\end{cases} \\
\forall i \in N,
\end{gathered}
$$

$\sum_{(i, n) \in \delta^{+}(i)} x_{n j} \leq 1 \quad \forall i \in N$,

$y_{i}-y_{j}+|N| \times x_{i j} \leq|N|-1 \quad \forall i, j \in N \backslash\{o, d\}, \quad i \neq j$,

$x_{i j} \in\{0,1\}, \quad \forall(i, j) \in A$,

$y_{i}$ real number $\forall i, j \in N \backslash\{o, d\}$,

where $x_{i j}$ takes the value of 1 if $(i, j)$ is a link in the tour, $\delta^{+}(i)$ is the set of all outgoing arcs of node $i$, and $\delta^{-}(i)$ is the set of all incoming arcs of node $i$.

Constraint (8) ensures that the total incoming flow entering a node is equal to the total outgoing flow. This constraint represents what is formally called a flow conservation constraint. At the same time, Constraint (9) guarantees that the outgoing degree of each node is at most one, while Constraint (10) is still necessary in order to guarantee the elementarily of the solution path and prevent sub-tours. Recall that a path is elementary if each node is visited at the most once. 
In the model given by Eqs. (7) to (12), the degree constraints, that is, Constraints (2) and (3), are removed and replaced by two weaker ones, namely Constraints (8) and (9). As a consequence, a feasible path does not need to be Hamiltonian, that is, visit each node exactly once.

To model our CBT problem, we propose a more general arc routing variant of the model represented by Eqs. (7) to (12). The proposed formulation is given by the following pure IP problem.

$\min \sum_{(i, j) \in A} c_{i j} \times x_{i j}$,

s.t.:

$$
\begin{gathered}
\sum_{(i, n) \in \delta^{+}(i)} x_{n j}-\sum_{(i, n) \in \delta^{-}(i)} x_{i n}= \begin{cases}-1 & \text { if } n=d \\
+1 & \text { if } n=o \\
0 & \text { if } n \in N \backslash\{o, d\}\end{cases} \\
\forall i \in N,
\end{gathered}
$$

$x_{i j}+x_{j i} \geq 1 \quad \forall(i, j) \in A^{\prime}$,

$y_{i}-y_{j}+|N| \times x_{i j} \leq|N|-1 \quad \forall i, j \in N \backslash\{o, d\}, \quad i \neq j$,

$x_{i j} \in\{0,1\} \quad \forall(i, j) \in A$,

$y_{i}$ non-negative integer $\forall i, j \in N \backslash\{o, d\}$,

where $x_{i j}$ takes the value of 1 if $(i, j)$ is a road traversed in the tour, $\delta^{+}(i)$ is the set of all outgoing roads of node $i$, $\delta^{-}(i)$ is the set of all incoming roads of node $i$, and $A^{\prime} \subset A$ denotes the set of all the roads representing PoI and pickup/dropout points.

The key difference between our formulation and the one provided by Eqs. (7) to (12) is clearly given by the relaxation of Constraint (9) by means of Constraint (15). While Constraint (9) requires that each node is visited at the most once, Constraint (15) simply ensures that each link representing a PoI (or a pickup/dropout point) is traversed at least once.

For the sake of completeness, let us outline the proof of the fact that the model given by Eqs. (13) to (18) always admits a solution corresponding to a minimal cost path. To see this, note that the network being strongly connected implies the existence of a feasible solution. At the same time, since the constraints and the objective function are all linear, the solution space is convex. Hence, the model given by Eqs. (13) to (18) admits global optimal solutions. In particular, since Constraint (16) eliminates the possibility of any sub-tour, there exists at least one path among the global optimal solutions which is also the shortest path from the origin node $o$ to the destination node $d$.
Note that Constraints (15) and (17) imply that $\forall(i, j) \in A^{\prime}, 0 \leq x_{i j}+x_{j i} \leq 2$, that is, the footprints of the city bus indicating the traffic flow through the different links in a minimal cost flow cannot be higher than 2. Since the optimal solution necessarily produces footprints between 0 and 2, there is just one residual graph and the augmenting paths are all minimal cost flows. Thus, we can efficiently augment the minimal cost network flow that solves the model given by Eqs. (13) to (18) into the three minimal cost sub-itineraries corresponding to the pickup stage (the bus picks up the tourists), the PoI visiting stage (the bus brings the tourists to visit all the PoI) and the dropoff stage (the bus drops off the tourists at their destinations).

As an example, let us consider a small network as shown in Fig. 6. All links are identical, that is, they are associated with the same traveling time $(\exists C$ s.t. $\forall(i, j) \in A$, $\left.c_{i j}=c_{j i}=C\right)$. The red colored links $(1,2),(3,2)$ and $(2,4)$ represent PoI and pickup/dropout points, and hence, they must be traversed at least once by the only city bus in service (remember the assumption that $q=1$ ).

After solving the problem as formulated above, the traffic flows (footprints) relative to the single links composing the minimal cost network flow are identified. In Fig. 6a, traffic flows $\left(x_{i j}+x_{j i}\right)$ have been used to label the links and delineate the footprints of the city bus. The value $q=1$ is also virtually shown using a dummy directional link from destination $d$ to origin $o$, to make the figure selfexplanatory.

As Fig. 6a shows, there exists a link not traversed by the city bus while there are two links that are traversed twice. Figure $6 \mathrm{~b}$ depicts the augmented minimal cost network, that is, the three directed paths corresponding to the three optimal sub-itineraries that the city bus must actually pursue to successfully complete its three-stage journey.

Note that a direct implementation of such a three-stage restriction in the mathematical formulation would be quite a tedious task and make the problem mathematically intractable. On the contrary, our formulation makes the problem tractable, the exact optimal solution reachable by using an optimization software such as GAMS, and the augmentation easily representable though three independent layers.

A more complex example showing the augmentation of a minimal cost network is provided in Fig. 7. In this figure, we show how to augment the artificial network reported in Fig. 4. As shown in Fig. 7, we split the three parts of the itinerary into three independent layers of the network. In the first layer (or first part), the city bus has a clear mandate to sweep through the links designated as pickup points and picks up all the tourists irrespective of any PoI or dropout point. This layer is connected to the second layer via some 


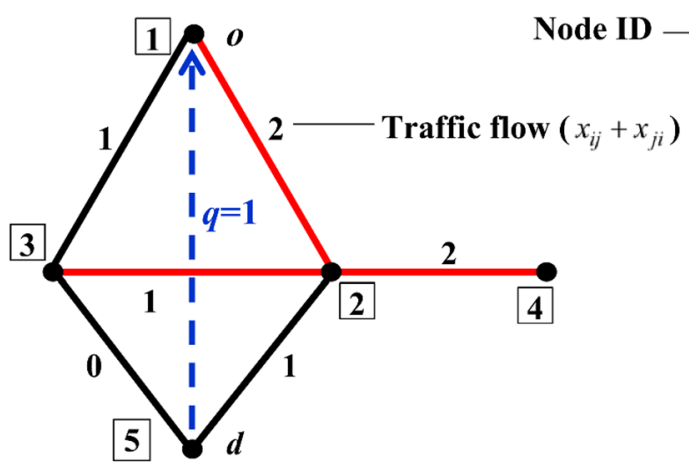

(a)

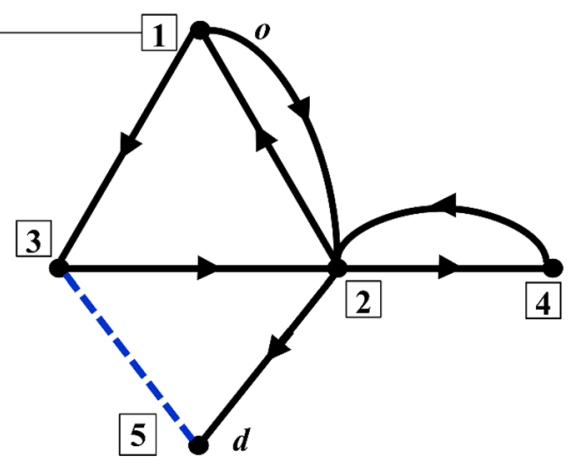

(b)

Fig. 6 A simple example: a footprints of the city bus (traffic flows) based on the minimum cost network flow on an undirected network (the red colored links represent places of interest and must be visited at least once); $\mathbf{b}$ the corresponding augmented directed network to show how the city bus navigates through the network

directional dummy links originating from the head or tail of the pickup links. In other words, when the city bus is done with getting all the tourists on board (the first layer), it descends through a dummy link (devised at the end of the last pickup link) to the second layer to visit the PoI. In a similar fashion, the city bus descends to the third layer once all the designated PoI have been visited (the second layer). Note that the dummy links serve merely to connect the layers and have zero traversing time.

The above example was encoded and solved using GAMS/CPLEX [65].

Summarizing, the methodology proposed for the CBT problem comprises the following steps:

- Step 1. Modeling the specific road network as a IP problem using Eqs. (13)-(18).

- Step 2. Solving the model given by Eqs. (13) to (18) using GAMS.

The GAMS code written for the problem returns (see also Sect. 5):

- the exact optimal solution identifying the footprints of the city bus relative to all the arcs forming the minimal cost network;

- the augmenting paths corresponding to the pickup stage, the PoI visiting stage and the drop-off stage.

\section{Numerical evaluations}

We have applied the methodology described in the previous section to a pilot study that involves the network of Melbourne's central region encompassing the central business district (CBD) and the surrounding area. This network, shown in Fig. 8, consists of 100 nodes and 362

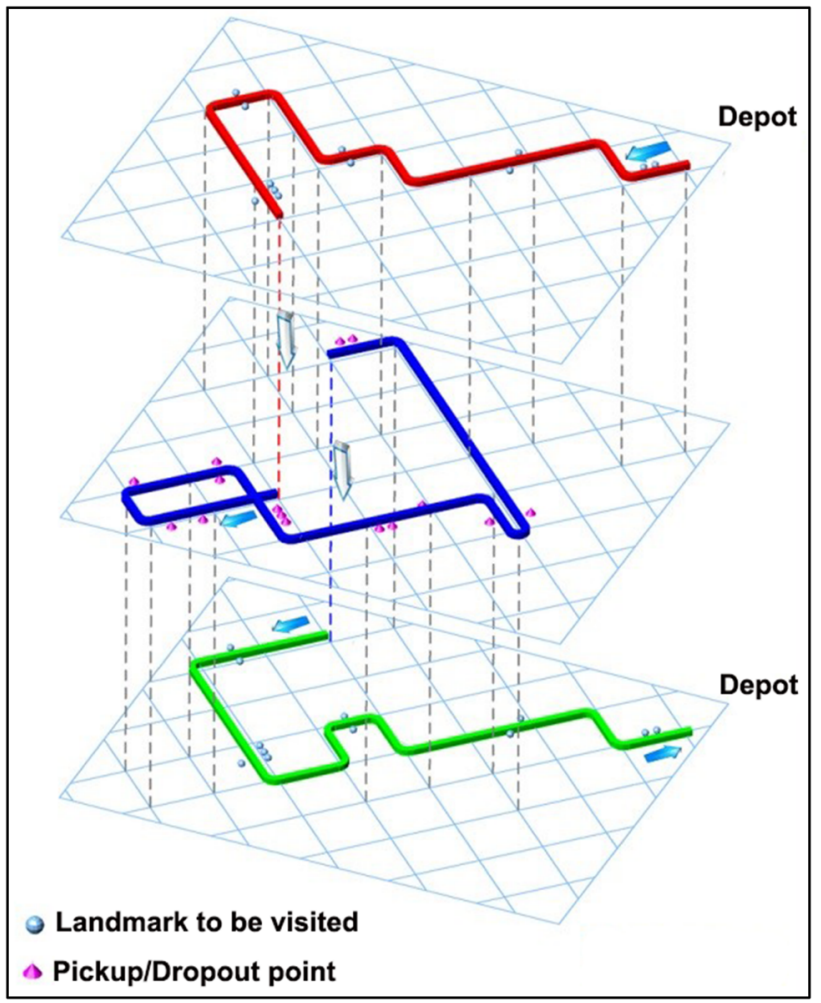

Fig. 7 Augmenting paths of the network in Fig. 4 in three independent layers

directional roads. For the ease of demonstration and data privacy, we changed the setup of the data and assumed node 83 located at the bottom right corner to be both the depot (or origin) and the destination node. The pilot study was conducted considering the 20 pickup/dropout links and the 8 PoI shown in Fig. 8.

Solving the optimal CBT problem with the proposed three-layer methodology, we obtain the detour depicted in 


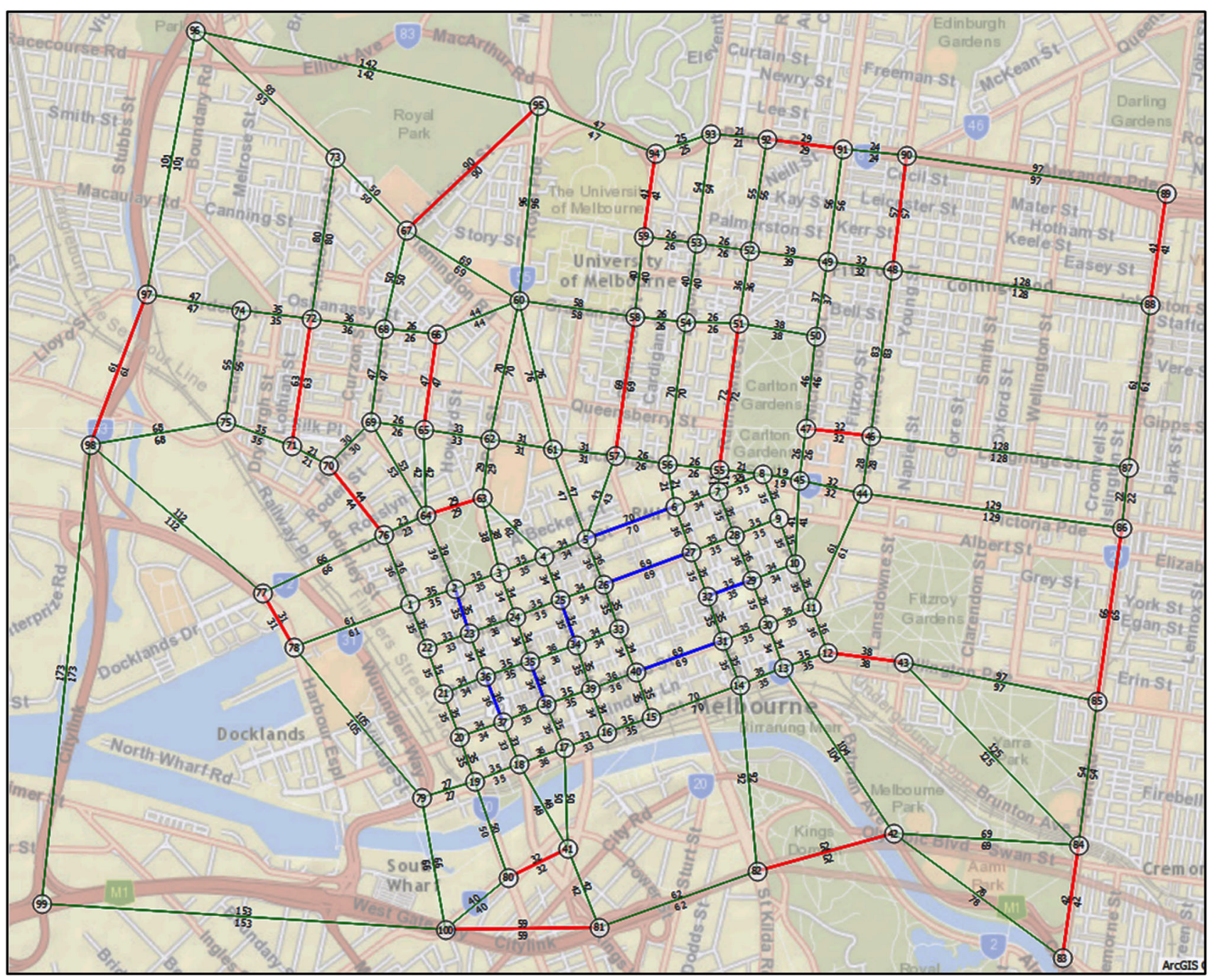

Fig. 8 Melbourne's central region: the red and blue colored links represent pickup/dropout points and places of interests, respectively. The numbers on the links indicate the traversing time (per 0.01 unit of time)

Fig. 9. The three parts of the optimal itinerary are distinctly shown by three different colors.

The GAMS code implemented to solve the CBT problem in the pilot study is included in "Appendix." The code developed for the current project is populated by an interface code accounting for the inputs received from the users. Using this code, the topography of the augmented network and traffic-related information are plugged into the GAMS code. In order to place the code in Appendix, we have cut off some of the lines, namely those pertaining to the topography of the network.

Regarding the performance of the DSS in solving the model, an issue of crucial importance for mobile apps and websites, note that all the computations relative to the initial tests are carried out using a standard desktop computer Intel(R) Core(TM) i7-4790 CPU @ $3.60 \mathrm{GHz}$ and
16.0 GB RAM. Based on the results obtained, inquiries of the clients (users) can be answered in a fraction of a minute. Nevertheless, the speed of computation could be greatly improved using parallel computation and employing a more advanced CPU power.

Finally, note that, in the initial tests, users are not completely free to choose the PoI to visit or the pickup/dropout points. Even though there is no restriction from a theoretical viewpoint on the possibility of choosing different PoI or pickup/dropout points (Eqs. 13-18) can easily accommodate these dynamic changes), for the initial deployment of the app, users are offered a limited number of packages consisting of a fixed number of PoI. However, the users can choose among several pickup/dropout points and undergo to different price tags depending on the proximity of the 


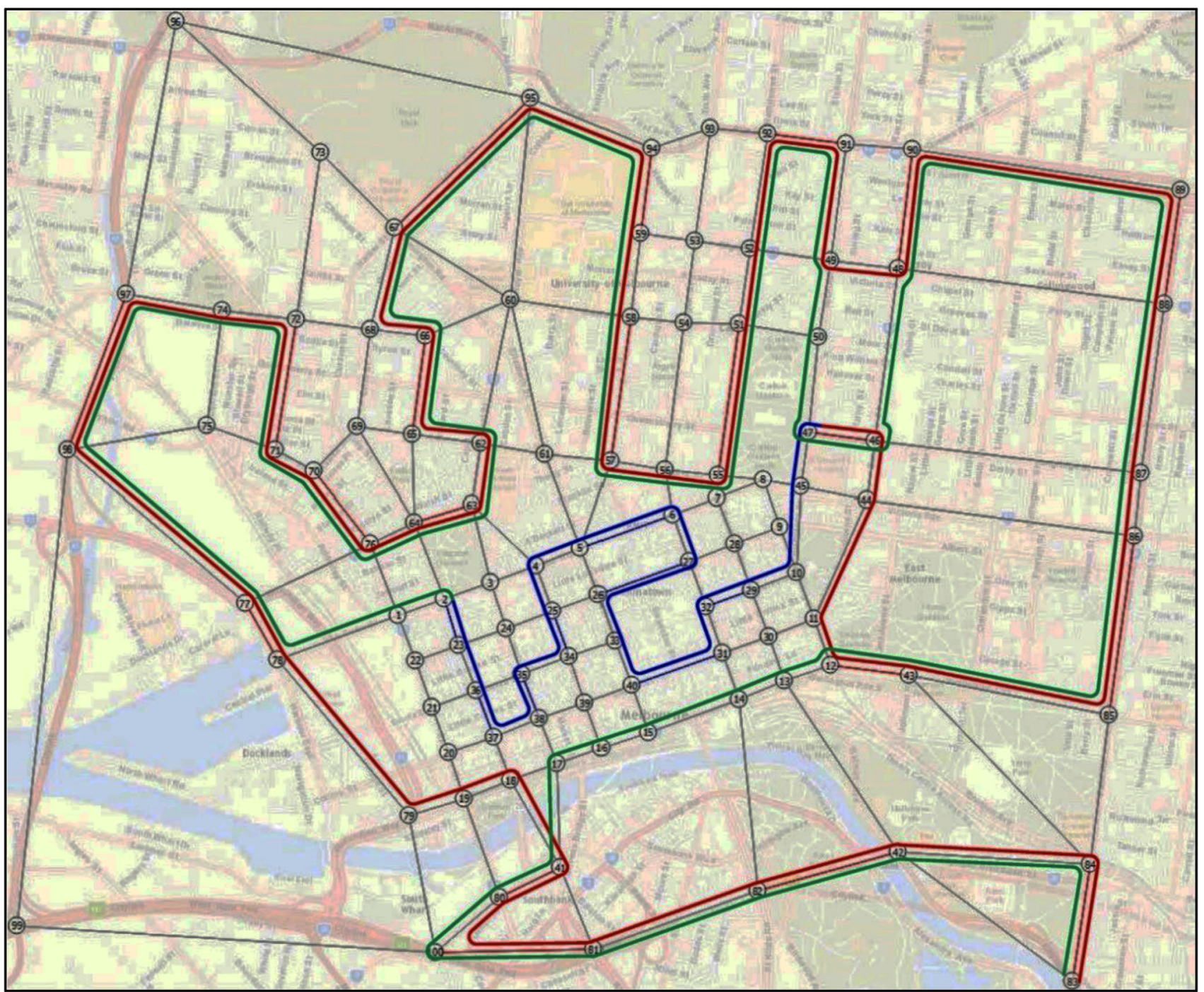

Fig. 9 Melbourne's central region and the optimal tour of a city bus serving 20 hotels (pickup points) and covering 8 landmarks (places of interests)

selected pickup/dropout points to the origin and destination points.

\section{Conclusion}

This study has presented a concrete attempt to promote tourism industry using ICT. In particular, it has shown how an advanced app and website can be developed using a CBT scheme, that is, a city bus service that allows tourists to visit the major landmarks in a short span of time.

First, we have investigated the role of ICT and, in particular, of the smartphone in the tourism industry in a broader sense. Hence, we have developed an efficient and smart way to plan the itinerary of a city bus catering to bus operators as well as tourists by minimizing operation (mileage) costs.

To the best of the authors' knowledge, no systematic DSS has been formulated or applied to the city bus system, even though it is far from being a new business. This study attempts to fill this knowledge gap. To this end, a mobile app and a website supported by GAMS (a leading optimization software) have been designed and are being developed to optimize the operations of the city bus business. The app/website offers a variety of tour options indicating a list of landmarks to be visited. The customers including hoteliers, travel agents and tourists can then book and purchase appropriate tour plans based on the seats available online. The customers are also required to indicate pickup/dropout locations which can be chosen from 
either a list of pre-planned stops or among places of their own convenience and choice. The choice of pickup/dropout places may be subject to an additional charge depending on the fleet availability, schedule of the itinerary and proximity. The app/website then delivers a confirmation note as well as an exact itinerary plan with departure and arrival times.

There are a number of lines of research for further investigation, such as dynamic pricing, fleet flexibility, customer satisfaction. The existing schemes-including the one developed in this research-are based on a fixed pricing rate, that is, the prices of tour options are fixed at all times irrespective of demand and fleet availability. It is of highest importance to develop a sophisticated, flexible and dynamic pricing mechanism (similar to those used for airline tickets) to carefully take both the demand and supply sides into consideration. This would greatly promote the business by accommodating long-term tourist demand.

In the city bus business, the main costs are represented by the fleet operation costs. Flexibility in the fleet operations can bring the costs down. Thus, the study of joint ventures across various bus operators is an important area for further investigation.

The ride sharing service (known as Uber) can also contribute to the tourism industry, in general, and to the city bus business, in particular. The integration of Uber as a primal or auxiliary service to city bus services is worthy of further studies.

Finally, since all businesses must aim at customer satisfaction, it is of highest importance to conduct market research to find out how to make city bus services more appealing to tourists.

Acknowledgement The authors would like to thank the anonymous reviewers and the editor for their insightful comments.

Open Access This article is distributed under the terms of the Creative Commons Attribution 4.0 International License (http:// creativecommons.org/licenses/by/4.0/), which permits unrestricted use, distribution, and reproduction in any medium, provided you give appropriate credit to the original author(s) and the source, provide a link to the Creative Commons license, and indicate if changes were made.

\section{Appendix: The GAMS code implemented to solve the proposed CBT problem in the pilot study}

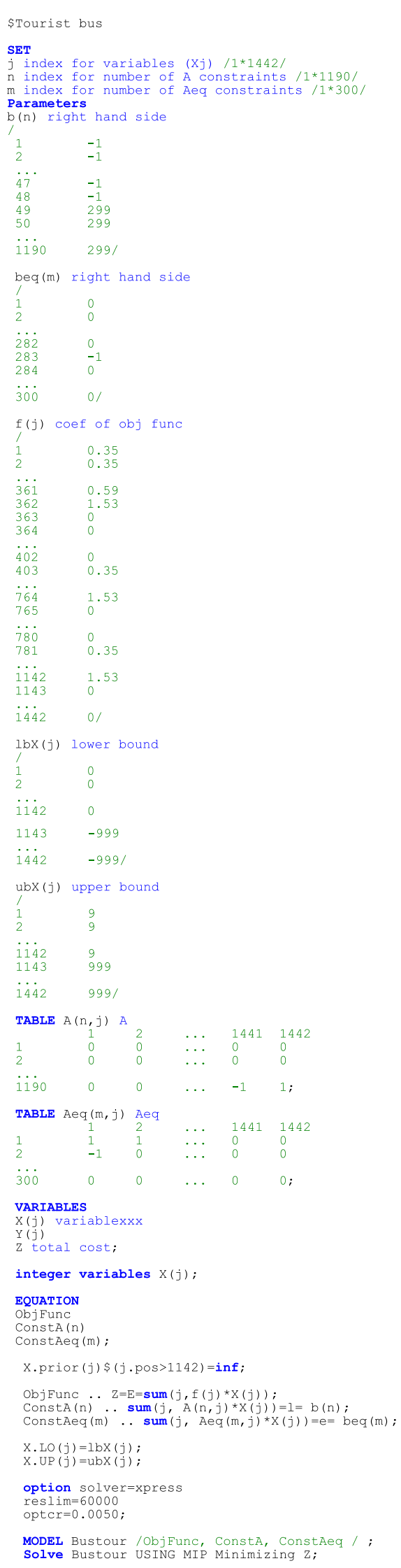




\section{References}

1. Buhalis D, Law R (2008) Progress in information technology and tourism management: 20 years on and 10 years after the Internet-the state of eTourism research. Tour Manag 29(4):609-623

2. Koo C, Joun Y, Han H, Chung N (2013) The impact of potential travellers' media cultural experiences. In: Xiang Z, Tussyadiah I (Eds.) Information and communication technologies in tourism 2014: proceedings of the international conference in Dublin, Ireland, January 21-24, 2014, Springer, Berlin, pp 579-592

3. Tussyadiah IP, Wang D (2014) Tourists' attitudes toward proactive smartphone systems. J Travel Res 55(4):493-508

4. Agag GM, El-Masry AA (2016) Why do consumers trust online travel websites? Drivers and outcomes of consumer trust toward online travel websites. J Travel Res 56(3):347-369

5. Wang X, Li XR, Zhen F, Zhang JH (2016) How smart is your tourist attraction?: Measuring tourist preferences of smart tourism attractions via a FCEM-AHP and IPA approach. Tour Manag 54:309-320

6. Okazaki S, Campo S, Andreu L, Romero J (2014) A latent class analysis of Spanish travelers' mobile internet usage in travel planning and execution. Cornell Hosp Q 56(2):191-201

7. Wang D, Xiang Z (2012) The new landscape of travel: a comprehensive analysis of smartphone apps. In: Fuchs M, Ricci F, Cantoni L (eds) Information and communication technologies in tourism. Springer, Wien, pp 308-319

8. Borràs J, Moreno A, Valls A (2014) Intelligent tourism recommender systems: a survey. Expert Syst Appl 41(16):7370-7389

9. Gavalas D, Kasapakis V, Konstantopoulos C, Pantziou G, Vathis $\mathrm{N}$, Zaroliagis $\mathrm{C}$ (2015) The eCOMPASS multimodal tourist tour planner. Expert Syst Appl 42(21):7303-7316

10. Oliveira CAS, Pardalos PM (2011) Mathematical aspects of network routing optimization. Springer optimization and its applications, vol 53. Springer, Berlin

11. Migdalas A, Sifaleras A, Georgiadis CK, Papathanasiou J, Stiakakis E (eds) (2013) Optimization theory, decision making, and operations research applications. Proceedings in mathematics \& statistics, vol. 31, Springer, New York

12. Papadimitriou CH (1977) The Euclidean travelling salesman problem is NP-complete. Theor Comput Sci 4(3):237-244

13. Tsiligirides $T$ (1984) Heuristic methods applied to orienteering. J Oper Res Soc 35(9):797-809

14. Golden BL, Levy L, Vohra R (1987) The orienteering problem. Nav Res Log 34(3):307-318

15. Eiselt HA, Gendreau M, Laporte G (1995) Arc routing problems, part II: the rural postman problem. Oper Res 43(3):399-414

16. Feillet D, Dejax P, Gendreau M (2005) Traveling salesman problems with profits. Transp Sci 39(2):188-205

17. Corberán A, Prins C (2010) Recent results on arc routing problems: an annotated bibliography. Networks 56(1):50-69

18. Pasqualetti F, Franchi A, Bullo F (2010) On optimal cooperative patrolling. In: 49th IEEE conference on decision and control

19. Vansteenwegen P, Souffriau W, Van Oudheusden D (2011) The orienteering problem: a survey. Eur J Oper Res 209(1):1-10

20. Chen M, Knecht S, Murphy HC (2015) An investigation of features and functions of smartphone applications for hotel chains. In: LENTER2015, Lugano, Switzerland

21. Wang D, Xiang Z, Law R, Ki TP (2016) Assessing hotel-related smartphone apps using online reviews. J Hosp Mark Manag 25(3):291-313

22. Miller CE, Tucker AW, Zemlin RA (1960) Integer programming formulation of traveling salesman problems. J ACM 7(4):326-329

23. MacKay K, Vogt C (2012) Information technology in everyday and vacation contexts. Ann Tour Res 39(3):1380-1401
24. Wang D, Xiang Z, Fesenmaier DR (2014) Adapting to the mobile world: a model of smartphone use. Ann Tour Res 48:11-26

25. Stienmetz JL, Levy SE, Boo S (2013) Factors influencing the usability of mobile destination management organization websites. J Travel Res 52(4):453-464

26. Jang SC (2005) The past, present, and future research of online information search. J Travel Tour Mark 17(2-3):41-47

27. Lubbe B, Louw L (2010) The perceived value of mobile devices to passengers across the airline travel activity chain. J Air Transp Manag 16(1):12-15

28. Wang H-Y, Wang S-H (2010) Predicting mobile hotel reservation adoption: insight from a perceived value standpoint. Int J Hosp Manag 29(4):598-608

29. Law R, Buhalis D, Cobanoglu C (2014) Progress on information and communication technologies in hospitality and tourism. Int $\mathrm{J}$ Contemp Hosp Manag 26(5):727-750

30. eMarketer (2015) How many smartphone users are officially addicted? http://www.emarketer.com/Article/How-Many-Smart phone-Users-Officially-Addicted/1012800

31. Deloitte (2015) Mobile consumer survey 2015-the Australian cut. http://landing.deloitte.com.au/rs/761-IBL-328/images/deloi tte-au-tmt-mobile-consumer-survey-2015-291015.pdf?mkt_tok=3 RkMMJWWfF9wsRokvaTIe+/hmjTEU5z16e8sXqSwhIkz2EFye +LIHETpodcMT8RqNr/YDBceEJhqyQJxPr3CKtEN09dxRhLg $\mathrm{AA}==$

32. Mang CF, Piper LA, Brown NR (2016) The incidence of smartphone usage among tourists. Int J Tour Res 18(6):591-601

33. Murphy HC, Chen M-M, Cossutta M (2016) An investigation of multiple devices and information sources used in the hotel booking process. Tour Manag 52:44-51

34. Anuar J, Musa M, Khalid K (2014) Smartphone's application adoption benefits using mobile hotel reservation system (MHRS) among 3 to 5-star city hotels in Malaysia. Proc Soc Behav Sci 130:552-557

35. Brown B, Chalmers M (2003) Tourism and mobile technology. In: ECSCW 2003

36. Souffriau W, Vansteenwegen P (2010) Tourist trip planning functionalities: state-of-the-art and future. Springer, Berlin

37. Deitch R, Ladany SP (2000) The one-period bus touring problem: solved by an effective heuristic for the orienteering tour problem and improvement algorithm. Eur J Oper Res 127(1):69-77

38. Deitch R, Ladany SP (2001) Determination of optimal one-period tourist bus tours with identical starting and terminal points. Int $\mathrm{J}$ Serv Technol Manag 2(1-2):116-129

39. Bolzoni P, Helmer S, Wellenzohn K, Gamper J, Andritsos $P$ (2014) Efficient itinerary planning with category constraints. In: Proceedings of the 22nd ACM SIGSPATIAL international conference on advances in geographic information systems, pp 203-212

40. Yu J, Aslam J, Karaman S, Rus D (2014) Optimal tourist problem and anytime planning of trip itineraries. arXiv preprint arXiv: 1409.853643

41. Brilhante I, Macedo JA, Nardini FM, Perego R, Renso C (2015) Planning sightseeing tours using crowdsensed trajectories. SIGSPATIAL Spec 7(1):59-66

42. Gavalas D, Konstantopoulos C, Mastakas K, Pantziou G, Vathis N (2015) Heuristics for the time dependent team orienteering problem: application to tourist route planning. Comput Oper Res 62:36-50

43. Lew A, McKercher B (2006) Modeling tourist movements: a local destination analysis. Ann Tour Res 33(2):403-423

44. Hensher DA, Greene WH, Ho CQ (2016) Random regret minimization and random utility maximization in the presence of preference heterogeneity: an empirical contrast. J Transp Eng 142(4):04016009 
45. Chorus CG (2012) Random regret minimization: an overview of model properties and empirical evidence. Transp Rev 32(1): 75-92

46. Chorus CG (2014) A generalized random regret minimization model. Transp Res B Methodol 68:224-238

47. Prato CG (2014) Expanding the applicability of random regret minimization for route choice analysis. Transportation 41(2): 351-375

48. Leong W, Hensher DA (2015) Contrasts of relative advantage maximisation with random utility maximisation and regret minimisation. J Transp Econ Policy 49(1):167-186

49. Gan H, Ye X (2014) Leave the expressway or not? Impact of dynamic information. J Mod Transp 22(2):96-103

50. Yue Y, Luo S, Luo T (2016) Micro-simulation model of two-lane freeway vehicles for obtaining traffic flow characteristics including safety condition. J Mod Transp 24(3):187-195

51. Gavalas D, Konstantopoulos C, Mastakas K, Pantziou G (2014) A survey on algorithmic approaches for solving tourist trip design problems. J Heuristics 20(3):291-328

52. Schaller R, Elsweiler D (2014) Itinerary recommenders: how do users customize their routes and what can we learn from them? In: Proceedings of the 5th information interaction in context symposium

53. Kramer R, Modsching M, Hagen KT (2006) A city guide agent creating and adapting individual sightseeing tours based on field trial results. Int J Comput Intell Res 2(2):191-206

54. Meng B, Kim M-H, Hwang Y-H (2015) Users and non-users of smartphones for travel: differences in factors influencing the adoption decision. Asia Pac J Tour Res 20(10):1094-1110
55. Wikipedia (2016) City sightseeing. https://en.wikipedia.org/wiki/ City_Sightseeing

56. Ayeh JK, Au N, Law R (2013) Do we believe in TripAdvisor? Examining credibility perceptions and online travelers' attitude toward using user-generated content. J Travel Res 52(4):437-452

57. Chevaleyre Y (2004) Theoretical analysis of the multi-agent patrolling problem. In: IEEE/WIC/ACM international conference on intelligent agent technology

58. Casbeer DW, Kingston DB, Beard RW, McLain TW (2006) Cooperative forest fire surveillance using a team of small unmanned air vehicles. Int J Syst Sci 37(6):351-360

59. Divsalar A, Vansteenwegen P, Cattrysse D (2013) A variable neighborhood search method for the orienteering problem with hotel selection. Int J Prod Econ 145(1):150-160

60. Moonen M, Cattrysse D, Van Oudheusden D (2007) Organising patrol deployment against violent crimes. Oper Res 7(3):401-417

61. Winston WL, Goldberg JB (2004) Operations research: applications and algorithms, vol 3. Duxbury Press, Boston

62. Dewil R, Vansteenwegen P, Cattrysse D, Van Oudheusden D (2015) A minimum cost network flow model for the maximum covering and patrol routing problem. Eur J Oper Res 247(1):27-36

63. Orman AJ, Williams HP (2007) A survey of different integer programming formulations of the travelling salesman problem. In: Kontoghiorghes EJ, Gatu C (eds) Optimisation, econometric and financial analysis. Springer, Berlin, pp 91-104

64. Taccari L (2016) Integer programming formulations for the elementary shortest path problem. Eur J Oper Res 252(1):122-130

65. GAMS (2014) GAMS Development Corporation, Washington, DC. Accessed 2014. http://www.gams.com 